\title{
Bistability in Online Social Networks
}

\author{
Kuang Xu*, Chi-Kin Chau ${ }^{\dagger}$, Victor O.K. Li* \\ *Dept. of Electrical \& Electronic Engg, The University of Hong Kong, Pokfulam, Hong Kong, China \\ ${ }^{\dagger}$ Computer Laboratory, University of Cambridge, Cambridge, United Kingdom
}

\begin{abstract}
Online social networking is an increasingly prominent social phenomenon that has motivated much interesting research in networking. This work studies the dynamic evolvement of online social networks by a simple stochastic model that captures the inter-dependent social interactions. We show that multiple stable states exist in the evolvement of online social networks, leading to fundamental ramifications to the structures of online social networks and their applications.
\end{abstract}

\section{INTRODUCTION}

Online social networking systems (e.g., Facebook, Twitter) have been hugely popular in the Internet. There are various studies to harness such systems for both social and commercial applications, such as viral campaigns, social games, and opportunistic advertising [1]. Online social network is a continually evolving large-scale system, whose behaviour motivates a number of fundamental studies to model and analyze their properties. These studies aim to shed light on the structures of online social networks for developing novel and useful applications based on these networks.

In this work, we address the fundamental question about the dynamics of social networks - What is the long-term structure of online social networks, under realistic inter-dependent social interactions on the formation and disappearance of social bonds among the participants? Our contribution is to offer a simple yet realistic model of online social networks, so as to demonstrate non-trivial and salient characteristics. Particularly, we analyze the dynamic behaviour of this model, and show that two stable states of online social networks can exist at different periods of evolvement: one with a densely connected structure, another with a sparsely connected structure.

The ramification is that if applications of online social networks depend critically on the connectivity, then such applications may experience highly different characteristics during the course of evolvement of online social networks. We remark that multiple stable states of dynamic systems have been observed in physics and in network routing, and it is related to the hysteresis phenomenon [2]. To the best of our knowledge, this work is the first study that establishes such interesting connection to the structure of online social networks.

\section{MOdEL AND ReSults}

\section{A. A Model of Online Social Networks}

We consider the following simple dynamic model of online social networks. There are various forms of contacts between every pair of persons (e.g. conversation, instant messages, email) which can indicate the levels of acquaintance and closeness between them. We aim to study the dynamics and interactions of such levels of acquaintance among people in online social networks.

In this model, we treat all forms of contacts as some generic units of contacts. Consider $N$ pairs of persons. Assume each unit of contacts is created spontaneously as a Poisson process of rate $\nu$. Each contact then becomes stale and lasts for a period of time exponentially distributed with mean 1 .

A salient feature in this model is its ability to capture the inter-dependent social interactions in online social networks. We suppose that there is an activity threshold $L$ for all persons. If there are more than $L$ units of contacts between a pair of persons, say $i$ and $j$, then one of $i$ and $j$, with equal probability, will randomly pick one other person, say $k$ to contact, subject to the constraint that $k$ must currently have at least one unit of contact with $i$ or $j$. In this case, one unit of contact will be added between $i$ and $k$, or one will be added between $j$ and $k$, and it will last for a random period of time distributed exponentially with mean 1 .

The triggering of a new contact with one other person corresponds to one of the fundamental tie-formation mechanisms in network sociology, namely, cyclic closure [3], which refers to the establishment of new interactions with one's network neighbors.

\section{B. Multiple Stationary Distributions in Large Regime}

We consider $N$ is large. In this case, we are able to characterise the stationary distribution of contacts. Let $n_{i}$ be the number of pairs with $i$ active (unexpired) contacts in $N$ pairs of persons, and let $x_{i}^{N}=\frac{n_{i}}{N}$ be the fraction of pairs. At time $t$, the state of social interactions is defined as a vector: $\left(x_{i}^{N}(t): i=0, \ldots, \infty\right)$. The property of online social network can be approximated by a continuous Markov chain with transition rates defined as:

1) Spontaneous formation of new contacts: For all $i$,

$\left(\ldots, x_{i}^{N}, x_{i+1}^{N}, \ldots\right) \rightarrow\left(\ldots, x_{i}^{N}-\frac{1}{N}, x_{i+1}^{N}+\frac{1}{N}, \ldots\right)$ at rate $\nu n_{i}$

2) Disappearance of stale contacts: For $i \geq 1$,

$\left(\ldots, x_{i}^{N}, x_{i+1}^{N}, \ldots\right) \rightarrow\left(\ldots, x_{i}^{N}+\frac{1}{N}, x_{i+1}^{N}-\frac{1}{N}, \ldots\right)$ at rate $i n_{i}$

3) Triggering of new contacts by social interactions: For all $i$, $\left(\ldots, x_{i}^{N}, x_{i+1}^{N}, \ldots, x_{j}^{N}, x_{j+1}^{N}, \ldots\right) \rightarrow$

$\left(\ldots, x_{i}^{N}-\frac{1}{N}, x_{i+1}^{N}+\frac{1}{N}, \ldots, x_{j}^{N}-\frac{1}{N}, x_{j+1}^{N}+\frac{1}{N}, \ldots\right)$ at rate $\frac{N-n_{0}}{N} \sum_{k=L}^{\infty} n_{k} \nu$

The derivations of such a continuous Markov chain follows the standard technique of approximating large interactive 


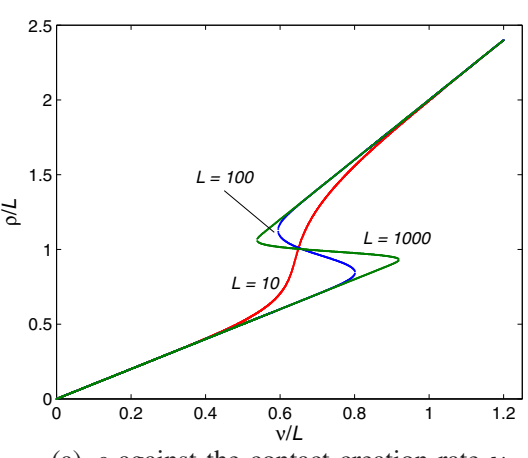

(a) $\rho$ against the contact creation rate $\nu$

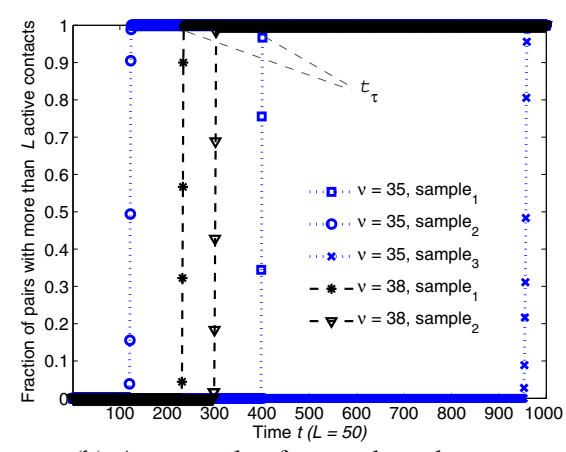

(b) An example of network evolvement

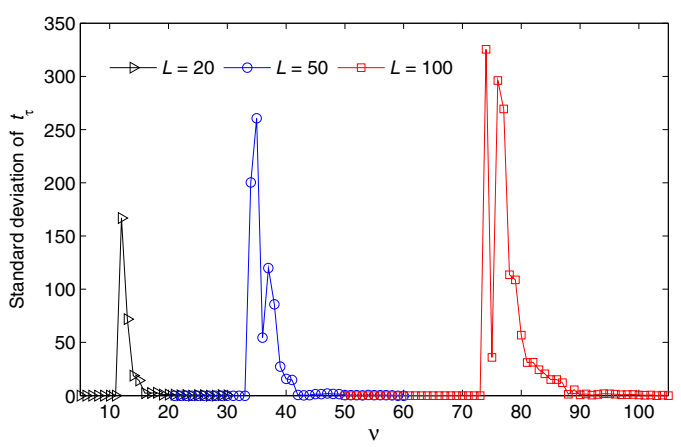

(c) Deviation of $t_{\tau}$

Fig. 1. Results

stochastic networks in [2]. We skip the detailed derivations because of the space constraint.

Applying Kurtz Theorem [4], when $N \rightarrow \infty$, the distribution of $x_{i}^{N}(t)$ converges to the limit $x_{i}(t)$ defined by the following differential equations:

$$
\frac{\mathrm{d} x_{0}(t)}{\mathrm{d} t}=x_{1}(t)-(\nu+\lambda(t)) x_{0}(t)
$$

and for $i \geq 1$,

$\frac{\mathrm{d} x_{i}(t)}{\mathrm{d} t}=(\nu+\lambda(t)) x_{i-1}(t)-(\nu+\lambda(t)+i) x_{i}(t)+(i+1) x_{i+1}(t)$

where $\lambda(t)=\nu\left(\sum_{k=L}^{\infty} x_{k}(t)\right)\left(1-x_{0}(t)\right)$.

To study the stationary distribution, we set $\frac{\mathrm{d} x_{i}(t)}{\mathrm{d} t}=0$. By the reversibility of the Markov chain, we obtain recursive equation for all $i \geq 1$ :

$$
(i+1) x_{i+1}=(\nu+\lambda) x_{i}
$$

where $\lambda=\nu\left(\sum_{k=L}^{\infty} x_{k}\right)\left(1-x_{0}\right)$.

We can solve Eqn. (1) for the stationary distribution $\left(x_{i}^{N}\right.$ : $i=0, \ldots, \infty)$ as follows.

Note that $\sum_{i=0}^{\infty} \frac{\rho^{i}}{i !}=e^{\rho}$. Let $\rho$ be the fixed point such that

$$
\rho=\nu+\nu\left(\sum_{i=L}^{\infty} \frac{\rho^{i}}{i !} e^{-\rho}\right)\left(1-e^{-\rho}\right)
$$

Then, one can check that the following stationary distribution solves Eqn. (1):

$$
x_{i}=\frac{\rho^{i}}{i !} e^{-\rho}
$$

Fig. 1(a) depicts the numerical relationship between $\rho$ and $\nu$ given certain $L$ in Eqn. (1). Note that Eqn. (2) may admit more than one fixed point for some values of $\nu$ and $L$. For instance, we found that $\rho=900.5,950.2$, or 1800 can numerically satisfy Eqn. (2) when $\nu=900$ and $L=1000$, of which only two are stable. Therefore, the implication is that bistability can exist, such that there can be two different stationary distributions. Note that different values of $\rho$ correspond to the different connectivities of online social networks. A larger $\rho$ means that there are more pairs of persons having larger number of contacts between them.

\section{Simulation}

Based on the model, we study empirically the evolvement of online social networks by simulation. Denote by $s_{i}$ the fraction of pairs of persons with more than $L$ active contacts, that is, $s_{i} \triangleq \sum_{i=L+1}^{\infty} x_{i}$. We study the saturation time $t_{\tau}$ at which $s_{i}$ grows to 1 . Fig. 1(b) shows the result based on a network of 100 nodes. We set the activity threshold $L=50$, and vary the contact creation rate $\nu$. We observe that under certain values of $\nu, t_{\tau}$ appears to have different values in different runs, for example, $t_{\tau}=236$ or 304 when $\nu=38$.

Furthermore, we specifically study the deviation of $t_{\tau}$. Fig. 1(c) shows the standard deviations of $t_{\tau}$ under different values of $L$. We can observe that, given $L, t_{\tau}$ appears almost the same under most of $\nu$ (i.e., zero standard deviation), but can be rather different in certain range of values of $\nu$ (i.e., large non-zero standard deviation). This indicates that $x_{i}$ can reach multiple stationary distributions given certain $\nu$ and $L$, which validates our analysis that multiple stable states can exist during the course of network evolvement.

\section{CONClusion ANd Future Work}

In this paper, we study the dynamic evolvement of online social networks and present a simple stochastic model that captures the inter-dependent social interactions. We show that the fraction of pairs of persons that are engaged in interactions may have multiple stationary distributions, according to different values of certain parameters. We are investigating the distribution of communities of persons that are engaged in interactions with each other and the transient evolution of social interactions in this model. We will also apply our model to various applications of online social networks (e.g., viral campaigns, social games and opportunistic advertising), and study the dynamic properties of these applications.

\section{REFERENCES}

[1] A. L. Montgomery, "Applying quantitative marketing techniques to the internet," Interfaces, vol. 31, pp. 90-108, 2001.

[2] R. J. Gibbens, P. J. Hunt, and F. P. Kelly, "Bistability in communication networks," Disorder in physical systems, pp. 113-128, 1990.

[3] G. Kossinets and D. J. Watts, "Empirical analysis of an evolving social network," SCIENCE, vol. 311, pp. 88-90, 2006.

[4] A. Shwartz and A. Weiss, Large deviations for performance analysis: queues, communication, and computing. Chapman \& Hall, 1995, ch. 5, pp. 75-84. 